\title{
Solvability of Fuzzy Relational Equations Employing Undefined Values
}

\author{
Nhung Cao \\ CE IT4I - IRAFM, University of Ostrava, \\ 30. dubna 22, 70103 Ostrava, Czech Republic, \\ nhung.cao@osu.cz
}

\begin{abstract}
The paper aims at studying the solvability of fuzzy relational equations where the input fuzzy sets may have undefined values. The appropriate operations dealing undefined values are taken from the algebras built in partial fuzzy set theory, in particular, the Bochvar operations, the Sobociński operations, the Kleene operations and the lower estimation operations are used for the investigation. We first elaborate new models using distinct operations handling undefined values and mirroring the shapes of the implicative model and Mamdani-Assilian model. Then the solvability criterions for such new systems of fuzzy relational equations are set up, and consequently, we show a way of checking whether the systems are solvable or not.
\end{abstract}

Keywords: Fuzzy relational equations, Undefined values, Partial fuzzy set theory.

\section{Introduction and Preliminaries}

\subsection{Introduction and motivation}

Systems of fuzzy relational equations were firstly studied by Sanchez [22]. During the years later, the topic has attracted the interest of several authors and has become an important topic in fuzzy mathematics, especially in fuzzy control and approximate reasoning. The most focused problem relating the topic is finding the solvability criterions for the systems of fuzzy relational equations. A number of extensive results have been achieved, regarding the unique positions of the implicative model and the Mamdani-Assilian model [19] among the solutions of the systems (cf. $[17,18,20])$, the Ruspini condition of the antecedent fuzzy sets (cf. [24]), and the continuous model of fuzzy rules [21]. Apart from the fuzzy control, the systems of fuzzy relational equations have been widely applied in many other areas covering the duality of optimization problems with generalized fuzzy relational equations [16], the linear optimization problems [15], or the posynomial geometric programming [25]. Up to the present time, the topic has been continuously updated both on theoretical and applied aspects, see $[12,8,13,5]$ for the most relevant results.

Standardly, there are two systems of fuzzy relational equations: the sup- $\mathrm{T}$ system represented by the form $A_{i} \circ R=B_{i}$ and the inf-R system represented by the form $A_{i} \triangleleft R=B_{i}$. In this work, we follow these settings, however, the antecedents $A_{i}$ will be partial fuzzy sets whose membership degrees can be undefined values. For the initial investigation, the consequents $B_{i}$ will be kept in the form of fully defined fuzzy sets. The idea of considering the occurrence of undefined values in the systems naturally stems from the formation of partial fuzzy logic and the so-called partial fuzzy set theory $[2,10,1,9]$, and the application of such topics into fuzzy relational compositions to model the compositions dealing with undefined values $[6,7]$. It is worth recalling that fuzzy partial logics are a generalization of three-valued logics into many-valued logics, usually $[0,1]$-valued logics and they model the vagueness phenomenon extended by the dummy value $\star$ representing undefined truth values. Several well-known threevalued logics handling undefined values have been generalized such as Bochvar logic, Sobociński logic, McCarthy logic, and Kleene logic (cf. [14, 4, 3]). In terms of computation, let us reduce the terms of logics to algebras of operations.

In the standard systems, the sup-T system is solvable if and only if the implicative model is its solution and the inf-R system is solvable if and only if the MamdaniAssilian model is its solution. We follow these results and thinking of whether similar results can be obtained for the new systems of equations dealing with undefined values appearing in the antecedents. In particular, we approach to answer the following questions: 
whether we can find appropriate models to build the solvability criterions for such the proposed systems, and in the positive case, which algebras of operations are used and do the models have similar shapes to the implicative model and Mamdani-Assilian model? Due to the variety of the algebras of operations dealing with undefined values, let us not choose all of them for the consideration but few best-known ones such as the Bochvar operations, the Sobociński operations, and the Kleene operations. Also, the new set of lower estimation operations designed for modeling one of the essential types of undefined values, i.e., missing values [7], is taken into account as well. Although this strategy of operations has been modified to form the Dragonfly algebra [23], it is the original idea, and we may apply it firstly, to observe its effect on the solvability of the new systems and to compare its use with the use of the three other ones.

\subsection{Systems of fuzzy relational equations}

Let us fix a complete residuated lattice $\mathcal{L}=$ $\langle[0,1], \wedge, \vee, \otimes, \rightarrow 0,1\rangle$ as the underlying algebraic structure. Moreover, by $\mathcal{F}(U)$ we denote the set of all fuzzy sets on a given universe $U$. Let $A_{i} \in \mathcal{F}(X), B_{i} \in$ $\mathcal{F}(Y), i=1, \ldots, m$ be the antecedent and consequent fuzzy sets, respectively. There are two standard systems of fuzzy relational equations considered with an unknown fuzzy relation $R \in \mathcal{F}(X \times Y)$ :

$$
\begin{aligned}
A_{i} \circ R & =B_{i}, \quad i=1,2, \ldots, m \quad(\text { sup- } \mathrm{T} \text { system }), \\
A_{i} \triangleleft R & =B_{i}, \quad i=1,2, \ldots, m \quad \text { (inf-R system), }
\end{aligned}
$$

where the used compositions (images) $\circ$ and $\triangleleft$ can be expanded as follows:

$$
\begin{aligned}
& \left(A_{i} \circ R\right)(y)=\bigvee_{x \in X}\left(A_{i}(x) \otimes R(x, y)\right), \\
& \left(A_{i} \triangleleft R\right)(y)=\bigwedge_{x \in X}\left(A_{i}(x) \rightarrow R(x, y)\right) .
\end{aligned}
$$

Two models with priority positions among other potential solutions of the systems are the MamdaniAssilian model (abbr. $\check{R}$ ) [19] and the implicative model (abbr. $\hat{R})$ :

$$
\begin{aligned}
\check{R}(x, y) & =\bigvee_{i=1}^{m}\left(A_{i}(x) \otimes B_{i}(y)\right), \\
\hat{R}(x, y) & =\bigwedge_{i=1}^{m}\left(A_{i}(x) \rightarrow B_{i}(y)\right) .
\end{aligned}
$$

The following theorem of the solvability criterion of the systems shows the priority of such models (cf. [11, $17,20])$.
Theorem 1.1. System $(1)[(2)]$ is solvable if and only if fuzzy relation $\hat{R}[\check{R}]$ is its solution and then $\hat{R}[\check{R}]$ is its greatest [least] solution.

\subsection{Distinct algebras of undefined values}

In this subsection, we briefly recall the Bochvar operations, the Sobociński operations, the Kleene operations and the lower estimation operations. Such the operations are defined on the support $L^{\star}=L \cup\{\star\}$ [2]. Note that the operations in the following definitions that are not attached by a subscript are taken from the structure $\mathcal{L}$.

Definition 1.1. [2] The Bochvar operation $c_{B} \in$ $\left\{\wedge_{B}, \vee_{B}, \otimes_{B}, \rightarrow_{B}\right\}, c_{B}: L^{\star} \times L^{\star} \rightarrow L^{\star}$ is represented by the following truth table:

\begin{tabular}{c|c|c|}
$c_{B}$ & $\beta$ & $\star$ \\
\hline$\alpha$ & $\alpha c \beta$ & $\star$ \\
\hline$\star$ & $\star$ & $\star$
\end{tabular}

Note that the dummy value $\star$ in this algebra works as the annihilator and whenever combined with another element, the operations return to $\star$ again.

Definition 1.2. [2] The Sobocinski operation $c_{S} \in$ $\left\{\wedge_{S}, \vee_{S}, \otimes_{S}\right\}, c_{S}: L^{\star} \times L^{\star} \rightarrow L^{\star}$ and the Sobocinski implication $\rightarrow_{S}: L^{\star} \times L^{\star} \rightarrow L^{\star}$ are represented by the following truth tables:

\begin{tabular}{c|c|c|}
$c_{S}$ & $\beta$ & $\star$ \\
\hline$\alpha$ & $\alpha c \beta$ & $\alpha$ \\
\hline$\star$ & $\beta$ & $\star$
\end{tabular},$\quad$\begin{tabular}{r|c|c|}
$\rightarrow_{S}$ & $\beta$ & $\star$ \\
\hline$\alpha$ & $\alpha \rightarrow \beta$ & $\neg \alpha$ \\
\hline$\star$ & $\beta$ & $\star$
\end{tabular}.

The dummy value $\star$ in this algebra employs as a sort of neutral element that is ignored by every another element in the support $L$. It should be noted that in the Bochvar algebra and the Sobociński algebra, the dummy value $\star$ is incomparable with any element from the support $L$. Thus, the ordering $\leq$ in these algebras only reflects the chain $L=[0,1]$ and that $\star \leq \star$.

Definition 1.3. [2] The Kleene operations $c_{K} \in$ $\left\{\wedge_{K}, \otimes_{K}\right\}, \vee_{K}$ and the Kleene implication $\rightarrow_{K}$ are binary operations on $L^{\star}$ represented by the following truth tables:

The dummy value $\star$ in the Kleene algebra works as in the Bochvar algebra on $L^{\star} \backslash\{0,1\}$ and it is comparable with 0 and 1 , i.e., $0 \leq \star \leq 1$.

Definition 1.4. [7] The lower estimation operations $c_{\ell} \in\left\{\otimes_{\ell}, \wedge_{\ell}\right\}, \vee_{\ell}, \rightarrow_{\ell}: L^{\star} \times L^{\star} \rightarrow L^{\star}$ are represented by the following truth tables: 


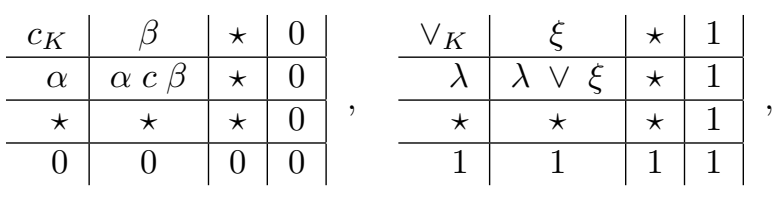

\begin{tabular}{r|c|c|c|}
$\rightarrow_{K}$ & $\xi$ & $\star$ & 1 \\
\hline$\alpha$ & $\alpha \rightarrow \xi$ & $\star$ & 1 \\
\hline$\star$ & $\star$ & $\star$ & 1 \\
\hline 0 & 1 & 1 & 1
\end{tabular}

\begin{tabular}{c|c|c|c|c|}
$c_{\ell}$ & $\beta$ & $\star$ & 0 & 1 \\
\hline$\alpha$ & $\alpha c \beta$ & $\star$ & 0 & $\alpha$ \\
\hline$\star$ & $\star$ & $\star$ & 0 & $\star$ \\
\hline 0 & 0 & 0 & 0 & 0 \\
\hline 1 & $\beta$ & $\star$ & 0 & 1
\end{tabular}

\begin{tabular}{|c|c|c|c|c|}
\hline$V_{\ell}$ & $\beta$ & $\star$ & 0 & 1 \\
\hline$\alpha$ & $\alpha \vee \beta$ & $\alpha$ & $\alpha$ & 1 \\
\hline * & $\beta$ & $\star$ & $\star$ & 1 \\
\hline 0 & $\beta$ & * & 0 & 1 \\
\hline 1 & 1 & 1 & 1 & 1 \\
\hline
\end{tabular}

\begin{tabular}{r|c|c|c|c}
$\rightarrow \ell$ & $\beta$ & $\star$ & 0 & 1 \\
\hline$\alpha$ & $\alpha \rightarrow \beta$ & $\star$ & $\neg \alpha$ & 1 \\
\hline$\star$ & $\beta$ & $\star$ & 0 & 1 \\
\hline 0 & 1 & 1 & 1 & 1 \\
\hline 1 & $\beta$ & $\star$ & 0 & 1
\end{tabular}.

On the set $L^{\star} \backslash\{0,1\}$, the lower estimation conjunction corresponds to the Bochvar conjunction, and the disjunction corresponds to the Sobociński disjunction. The implication behaves like the Sobociński implication when the first argument is $\star$ and like the Bochvar implication when the second argument is $\star$. The lower estimation operations applying for $\star$ and $\{0,1\}$ do preserve the ordering $0 \leq \star \leq 1$ as in the Kleene algebra.

In addition to those operations, we recall two useful external unary operations.

Definition 1.5. [2] The external operations $\downarrow, \uparrow$ : $L^{\star} \rightarrow L^{\star}$ are represented as follows: $\downarrow \alpha=0$ if $\alpha=\star$ and $\downarrow \alpha=\alpha$ otherwise; $\uparrow \alpha=1$ if $\alpha=\star$ and $\uparrow \alpha=\alpha$ otherwise.

\section{Systems of fuzzy relational equations employing undefined values}

\section{$2.1 \quad$ Sup-T system}

In the sequel, we use the notation $\mathcal{F}^{\star}(X)$ for the set of all partial fuzzy sets defined on $X$. Now, let us first consider the use of the lower estimation operations into the system. In particular, we approach to find the solvability of system

$$
A_{i} \circ_{\ell} R=B_{i}, \quad i=1, \ldots, m
$$

where $A_{i} \in \mathcal{F}^{\star}(X), B_{i} \in \mathcal{F}(Y)$ and $R \in \mathcal{F}^{\star}(X \times Y)$ is unknown.

In a similar shape with the implication model $\hat{R}$, we define model $\hat{R}_{\ell}$ :

$$
\hat{R}_{\ell}(x, y)=\bigwedge_{i=1}^{m}\left(\downarrow A_{i}(x) \rightarrow_{\ell} B_{i}(y)\right) .
$$

By this definition, $\hat{R}_{\ell}$ is a fuzzy relation on $X \times Y$. In order to study properties of $\hat{R}_{\ell}$, we need the following lemma:

Lemma 2.1. The following hold for any $a, b, c \in L^{\star}$ :

$$
\begin{aligned}
& \downarrow\left(a \otimes_{\ell} b\right) \leq c \quad \text { iff } \quad \downarrow a \leq \downarrow b \rightarrow_{\ell} c, \\
& \downarrow\left(a \vee_{\ell} b\right)=(\downarrow a) \vee_{\ell}(\downarrow b), \\
& \uparrow\left(a \rightarrow_{\ell} b\right) \geq c \quad \text { iff } \quad \uparrow b \geq \uparrow a \otimes_{\ell} c, \\
& a \rightarrow_{\ell}\left(\uparrow a \otimes_{\ell} b\right) \geq b .
\end{aligned}
$$

Sketch of the proof: Applying the definition of the lower estimation operations and the two external operations $\downarrow, \uparrow$, one can check the validity of (7)-(10) by replacing at least one argument by $\star$.

It should be recalled that when considering an inclusion of two partial fuzzy sets $B_{1}$ and $B_{2}$ on $Y$, we keep in mind the standard fuzzy subset defined as follows:

$$
B_{1} \subseteq B_{2} \quad \text { if } \quad B_{1}(y) \leq B_{2}(y), \forall y \in Y .
$$

\section{Theorem 2.2.}

$$
\downarrow\left(A_{i} \circ_{\ell} \hat{R}_{\ell}\right) \subseteq B_{i} .
$$

Sketch of the proof: For all $y \in Y$,

$$
\begin{aligned}
& \left(A_{i} \circ_{\ell} \hat{R}_{\ell}\right)(y) \\
& =\bigvee_{\ell \in X}\left(A_{i}(x) \otimes_{\ell} \bigwedge_{j=1}^{m}\left(\downarrow A_{j}(x) \rightarrow_{\ell} B_{j}(y)\right)\right) \\
& \leq \bigvee_{\ell \in X}\left(A_{i}(x) \otimes_{\ell}\left(\downarrow A_{i}(x) \rightarrow_{\ell} B_{i}(y)\right)\right) .
\end{aligned}
$$

Let $M(x)=A_{i}(x) \otimes_{\ell}\left(\downarrow A_{i}(x) \rightarrow_{\ell} B_{i}(y)\right)$ and let us consider two cases of $B_{i}(y)$ for $y \in Y$. In case of $B_{i}(y)=0, M(x)=\star$ if $A_{i}(x)=\star$ and $M(x)=0$ if $A_{i}(x) \neq \star$. Thus, $\downarrow \bigvee_{\ell x \in X} M(x)=\downarrow \star=0$ which is equal to $B_{i}(y)$. Now we consider the case of $B_{i}(y)>$ 0 . We have $M(x)=0$ if $A_{i}(x)=0$ and $M(x)=\star$ 
if $A_{i}(x)=\star$. If $A_{i}(x) \neq\{0, \star\}$ then based on the property that $a \otimes(a \rightarrow b) \leq b$ we imply that $M(x) \leq$ $B_{i}(y)$. Thus, $\downarrow \bigvee_{\ell x \in X} M(x)$ is always smaller than or equal to $B_{i}(y)$.

Corollary 2.3. For each $i=1, \ldots, m$, if there exists $x \in X$ such that $A_{i}(x) \neq\{0, \star\}$ and $B_{i}(y)>0$ for all $y \in Y$ then the following inclusion holds

$$
A_{i} \circ_{\ell} \hat{R}_{\ell} \subseteq B_{i}
$$

Sketch of the proof: Property (12) is proved based on the proof of (11). Indeed, based on the assumption of the existence of an $x \in X$ such that $A_{i}(x) \neq\{0, \star\}$ and $B_{i}(y)>0$ for all $y \in Y$, we can find $x^{\prime} \in X$ such that $A_{i}\left(x^{\prime}\right) \neq\{0, \star\}$ and $\bigvee_{\ell x \in X}\left(A_{i}(x) \otimes_{\ell}\left(\downarrow A_{i}(x) \rightarrow_{\ell} B_{i}(y)\right)\right)=A_{i}\left(x^{\prime}\right) \otimes_{\ell}$ $\left(\downarrow A_{i}\left(x^{\prime}\right) \rightarrow_{\ell} B_{i}(y)\right)$ which is different from $\{0, \star\}$ and smaller than or equal to $B_{i}(y)$.

Theorem 2.4. If system (5) is solvable then

$$
A_{i} \circ_{\ell} \hat{R}_{\ell} \supseteq B_{i} \text {. }
$$

Sketch of the proof: Using (7) and (8) the following sequence of the implications holds:

$$
\begin{aligned}
& \left(A_{i} \circ_{\ell} R\right)(y) \leq B_{i}(y), y \in Y \\
& \downarrow \bigvee_{\ell \in X}\left(A_{i}(x) \otimes_{\ell} R(x, y)\right) \leq B_{i}(y), y \in Y, \forall i \\
& \downarrow\left(A_{i}(x) \otimes_{\ell} R(x, y)\right) \leq B_{i}(y), x \in X, y \in Y, \forall i \\
& \downarrow R(x, y) \leq \downarrow A_{i}(x) \rightarrow_{\ell} B_{i}(y), x \in X, y \in Y, \forall i \\
& \downarrow R(x, y) \leq \bigwedge_{i=1}^{m}\left(\downarrow A_{i}(x) \rightarrow_{\ell} B_{i}(y)\right), x \in X, y \in Y .
\end{aligned}
$$

Thus, $\downarrow R \subseteq \hat{R}_{\ell}$ which implies $\left(A_{i} \circ_{\ell} \downarrow R\right) \subseteq$ $\left(A_{i} \circ_{\ell} \hat{R}_{\ell}\right)$.

Since $A_{i} \circ_{\ell} R=B_{i}$ is a fuzzy set on $Y$ whose membership degrees are fully defined, we can prove that $A_{i} \circ_{\ell} R=A_{i} \circ_{\ell} \downarrow R$. Hence

$$
B_{i} \subseteq\left(A_{i} \circ_{\ell} R\right) \subseteq\left(A_{i} \circ_{\ell} \hat{R}_{\ell}\right) .
$$

Theorem 2.5. For each $i=1, \ldots, m$, if there exists $x \in X$ such that $A_{i}(x) \neq\{0, \star\}$ and $B_{i}(y)>0$ for all $y \in Y$ then system (5) is solvable if and only if $\hat{R}_{\ell}$ is its solution. Moreover, in case of solvability, $\downarrow R \subseteq \hat{R}_{\ell}$ for any solution $R$ of the system.

Sketch of the proof: The proof is directly derived from Corollary 2.3 and Theorem 2.4.
Remark 2.1. The properties of model $\hat{R}_{\ell}$ for system (5) are given in inclusions (11), (12) and (13). Property (11) provides the general relation of the inferred outputs $A_{i} \circ_{\ell} \hat{R}_{\ell}$ and the consequents $B_{i}$, that they can be incomparable only at the values $y \in Y$ such that $\left(A_{i} \circ_{\ell} \hat{R}_{\ell}\right)(y)=\star$. Property (13) shows that in case of solvability of system (5), the inferred outputs are greater than or equal to the consequents and moreover, model $\hat{R}_{\ell}$ is always greater than or equal to $\downarrow R$ for any solution $R$ of the system. This property may help in narrowing the set of solutions, serving to find an approximate solution of system (5). It is trivial that both properties (11) and (13) are not sufficient to imply the solvability criterion of the system. However, if the assumptions presented in Corollary 2.3 are taken into account, then (12) holds, and the combination of (12) and (13) directly implies the solvability of the system. The assumption on the antecedents that there is at least $x \in X$ so that $A_{i}(x) \neq\{0, \star\}$ can be achieved easily since the fuzzy inputs usually contain membership degrees different from 0 and 1. In contrast, the assumption on the consequents that $B_{i}(y)$ has to be greater than 0 for all $y \in Y$ is rather strict, but it can be satisfied. In case of both assumptions hold, the solvability criterion of system (5) is set up (Theorem 2.5). There, to check the solvability of (5), it is sufficient to check whether $\hat{R}_{\ell}$ is its solution or not. In the case of negative, the system is not solvable.

Now, let us consider the use of the Sobociński operations i.e., considering system

$$
A_{i} \circ_{S} R=B_{i}, \quad i=1, \ldots, m .
$$

The model according to this algebra of operations is given by

$$
\hat{R}_{S}(x, y)=\bigwedge_{i=1}^{m}\left(\downarrow A_{i}(x) \rightarrow_{S} B_{i}(y)\right) .
$$

The properties presented in the following lemma are necessary for investigating properties of model $\hat{R}_{S}$.

Lemma 2.6. The following hold for any $a, b, c \in L^{\star}$ :

$$
\begin{aligned}
& \downarrow\left(a \otimes_{S} b\right) \leq c \quad \Rightarrow \quad \downarrow a \leq \downarrow b \rightarrow_{S} c, \\
& \downarrow\left(a \vee_{S} b\right)=(\downarrow a) \vee_{S}(\downarrow b), \\
& \uparrow\left(a \rightarrow_{S} b\right) \geq c \Rightarrow \uparrow b \geq \uparrow a \otimes_{S} c, \\
& \uparrow\left(a \wedge_{S} b\right)=(\uparrow a) \wedge_{S}(\uparrow b), \\
& a \rightarrow S\left(\uparrow a \otimes_{S} b\right) \geq b .
\end{aligned}
$$

Sketch of the proof: The proof is based on the definitions of the Sobociński operations and the operations $\downarrow, \uparrow$.

If we take into account Lemma 2.1 we may see that properties (16) and (18) preserve (7) and (9) respectively only in a weak form of the implications. 


\section{Theorem 2.7.}

$$
\left(\downarrow A_{i}\right) \circ_{S} \hat{R}_{S} \subseteq B_{i} .
$$

Sketch of the proof: For all $y \in Y$,

$$
\begin{aligned}
& \left(\left(\downarrow A_{i}\right) \circ_{S} \hat{R}_{S}\right)(y) \\
& =\bigvee_{x \in X}\left(\left(\downarrow A_{i}(x)\right) \otimes_{S} \bigwedge_{j=1}^{m}\left(\downarrow A_{j}(x) \rightarrow_{S} B_{j}(y)\right)\right) \\
& \leq \bigvee_{x \in X}\left(\left(\downarrow A_{i}(x)\right) \otimes_{S}\left(\downarrow A_{i}(x) \rightarrow_{S} B_{i}(y)\right)\right) .
\end{aligned}
$$

Let $M(x)=\left(\downarrow A_{i}(x)\right) \otimes_{S}\left(\downarrow A_{i}(x) \rightarrow_{S} B_{i}(y)\right)$. In case of $A_{i}(x) \in\{0, \star\}$, we get $M(x)=0$ which is always smaller than or equal to $B_{i}(y)$. In case of $A_{i}(x) \neq$ $\{0, \star\}, M(x)$ is a truth value in $L$ and it is trivial smaller than or equal to $B_{i}(y)$.

Theorem 2.8. If system (14) is solvable then

$$
A_{i} \circ_{S} \hat{R}_{S} \supseteq B_{i} .
$$

Sketch of the proof: Using (16)-(17) we can prove (22) in a similar way to the proof of (13). In particular, we first prove $\downarrow R \subseteq \hat{R}_{S}$. Then, since $A_{i} \circ_{S} R=B_{i}$ is a fuzzy set on $Y$ whose membership degrees are fully defined, we may prove that for $i=1, \ldots, m$ and $y \in Y$, $\left(A_{i} \circ_{S} R\right)(y)$ is greater than or equal to $\left(A_{i} \circ_{S} \downarrow R\right)(y)$. In case of the equality, it can be easily implied that $B_{i}(y) \leq\left(A_{i} \circ_{S} \hat{R}_{S}\right)(y)$. In case of the inequality, we may directly prove that $\left(A_{i} \circ_{S} R\right)(y) \leq\left(A_{i} \circ_{S} \hat{R}_{S}\right)(y)$ and thus, $B_{i}(y) \leq\left(A_{i} \circ_{S} \hat{R}_{S}\right)(y)$.

Remark 2.2. When using the Sobocinski operations, we do not obtain the solvability criterion for system (14). This fact is understandable due to the appearing of undefined values in the antecedents which may cause a strict inequality of the inferred outputs and the consequents. Indeed, one may refer from the proof of (21) that in case of without applying the operation $\downarrow$ to the antecedents, it may happen that the inferred output is equal to 1 at a certain $y \in Y, i \in\{1, \ldots, m\}$ which is possibly greater than $B_{i}(y)$. However, inclusion (22) remains as a good result for the application of the Sobocinski operations. Moreover, the proof of such inclusion shows that in case of solvability of system (14), $\downarrow R \subseteq \hat{R}_{S}$ for any solution $R$ of the system.

In the application of the Bochvar operations, one may observe that system

$$
A_{i} \circ_{B} R=B_{i}, \quad i=1, \ldots, m
$$

is not solvable. Indeed, whenever there is an $x \in X$ such that $A_{i}(x)=\star,\left(A_{i} \circ_{B} R\right)(y)$ will be equal to $\star$ for any $y \in Y$ which is incomparable to $B_{i}(y) \in L$.

When employing the Kleene operations, system

$$
A_{i} \circ_{K} R=B_{i}, \quad i=1, \ldots, m
$$

is possibly solvable. This happens only in two cases: when $\left(A_{i} \circ_{K} R\right)(y) \neq \star$ and when $\left(A_{i} \circ_{K} R\right)(y)=1$ for all $y \in Y$. The first case corresponds to considering $R$ so that $R(x, y)=0$ at the values $x \in X$ such that $A_{i}(x)=\star$, and $R(x, y) \neq \star$ at the values $x \in X$ such that $A_{i}(x) \neq 0$. The second case corresponds to the existence of an $x \in X$ such that $A_{i}(x)=1$ and $R(x, y)=1$. If model

$$
\hat{R}_{K}(x, y)=\bigwedge_{i=1}^{m}\left(\downarrow A_{i}(x) \rightarrow_{K} B_{i}(y)\right)
$$

is taken into account then the second case causes $B_{i}(y)=1$ for $i=1, \ldots, m$ and for all $y \in Y$. Such output fuzzy sets $B_{i}$ are usually not considered for fuzzy inference problems.

\subsection{Inf-R system}

In this subsection, we study the solvability of the inf$\mathrm{R}$ system dealing with undefined values in the antecedents. Let us again apply the lower estimation operations first. We have the following system

$$
A_{i} \triangleleft_{\ell} R=B_{i}, \quad i=1, \ldots, m
$$

where $A_{i} \in \mathcal{F}^{\star}(X), B_{i} \in \mathcal{F}(Y)$ and $R \in \mathcal{F}^{\star}(X \times Y)$ is unknown.

In a similar shape to the Mamdani-Assilian model $\check{R}$, we define the following model $\check{R}_{\ell}$ using the external operation $\uparrow$ :

$$
\check{R}_{\ell}(x, y)=\bigvee_{i=1}^{m}\left(\uparrow A_{i}(x) \otimes_{\ell} B_{i}(y)\right) .
$$

\section{Theorem 2.9.}

$$
A_{i} \triangleleft_{\ell} \check{R}_{\ell} \supseteq B_{i} .
$$

Sketch of the proof: Based on property (10) and the fact that $a \leq b$ for $a, b \in L$ implies $c \rightarrow_{\ell} a \leq c \rightarrow_{\ell} b$ for $c \in L^{\star}$, we obtain

$$
\begin{aligned}
& \left(A_{i} \triangleleft_{\ell} \check{R}_{\ell}\right)(y) \\
& \geq \bigwedge_{x \in X}\left(A_{i}(x) \rightarrow_{\ell}\left(\uparrow A_{i}(x) \otimes_{\ell} B_{i}(y)\right)\right) \geq B_{i}(y) .
\end{aligned}
$$


Theorem 2.10. If system (26) is solvable then

$$
A_{i} \triangleleft_{\ell} \check{R}_{\ell} \subseteq B_{i}
$$

Sketch of the proof: Applying property (9), the assumption that system $(26)$ is solvable, and the fact that $\uparrow\left(a \wedge_{\ell} b\right)=(\uparrow a) \wedge_{\ell}(\uparrow b)$ when $a \wedge_{\ell} b \neq \star$, the following sequence of the implications holds:

$$
\begin{aligned}
& \left(A_{i} \triangleleft_{\ell} R\right)(y) \geq B_{i}(y), y \in Y \\
& \uparrow \bigwedge_{x \in X}\left(A_{i}(x) \rightarrow_{\ell} R(x, y)\right) \geq B_{i}(y), y \in Y, \forall i \\
& \uparrow\left(A_{i}(x) \rightarrow_{\ell} R(x, y)\right) \geq B_{i}(y), x \in X, y \in Y, \forall i \\
& \uparrow R(x, y) \geq \uparrow A_{i}(x) \otimes_{\ell} B_{i}(y), x \in X, y \in Y, \forall i \\
& \uparrow R(x, y) \geq \bigvee_{\ell=1}^{m}\left(\uparrow A_{i}(x) \otimes_{\ell} B_{i}(y)\right), x \in X, y \in Y \\
& \\
& \uparrow R(x, y) \geq \check{R}_{\ell}(x, y), x \in X, y \in Y .
\end{aligned}
$$

Thus, $\uparrow R \supseteq \check{R}_{\ell}$ which implies $\left(A_{i} \triangleleft_{\ell} \uparrow R\right) \supseteq$ $\left(A_{i} \triangleleft_{\ell} \check{R}_{\ell}\right)$.

Based on the assumption that $\left(A_{i} \triangleleft_{\ell} R\right)(y)=B_{i}(y)$ is a value in $L$, we may prove that $A_{i} \triangleleft_{\ell} \uparrow R=A_{i} \triangleleft_{\ell} R$. Hence,

$$
B_{i} \supseteq\left(A_{i} \triangleleft_{\ell} R\right) \supseteq\left(A_{i} \triangleleft_{\ell} \check{R}_{\ell}\right) .
$$

Theorem 2.11. System (26) is solvable if and only if $\check{R}_{\ell}$ is its solution, and moreover, $\uparrow R \supseteq \check{R}_{\ell}$ for any solution $R$ of the system.

Sketch of the proof: The proof is directly derived from Theorem 2.9 and Theorem 2.10.

Remark 2.3. Unlike to system (5), the solvability criterion of system (26) can be easily achieved without any constraint on the antecedents and consequents. Such criterion is similar to the one presented in Theorem 1.1, which shows that model $\check{R}_{\ell}$ posses a unique position among other potential solutions of system (26). Thus, to check the solvability of the system, it is sufficient to check whether $\check{R}_{\ell}$ is its solution or not, and in case of negative, the system is not solvable. Such model $\check{R}_{\ell}$ ensures the solvability of the system.

Now, we investigate the solvability of the inf-R system with the application of the Sobocinski operations, the Bochvar operations and the Kleene operations. The systems are given as follows

$$
\begin{array}{ll}
A_{i} \triangleleft_{S} R=B_{i}, & i=1, \ldots, m, \\
A_{i} \triangleleft_{B} R=B_{i}, & i=1, \ldots, m, \\
A_{i} \triangleleft_{K} R=B_{i}, & i=1, \ldots, m .
\end{array}
$$

The models according to those algebras of operations are given as follows

$$
\begin{aligned}
\check{R}_{S}(x, y) & =\bigvee_{i=1}^{m}\left(\uparrow A_{i}(x) \otimes_{S} B_{i}(y)\right), \\
\check{R}_{B}(x, y) & =\bigvee_{i=1}^{m}\left(\uparrow A_{i}(x) \otimes_{B} B_{i}(y)\right), \\
\check{R}_{K}(x, y) & =\bigvee_{i=1}^{m}\left(\uparrow A_{i}(x) \otimes_{K} B_{i}(y)\right) .
\end{aligned}
$$

The question is, in the case of solvability, whether such models are solutions of systems (30)-(32), respectively, or not. As we may see, the answer is rather positive for the use of the Sobociński operations.

Theorem 2.12.

$$
A_{i} \triangleleft_{S} \check{R}_{S} \supseteq B_{i}
$$

Sketch of the proof: The proof is analogous to the proof of Theorem 2.9 i.e., it is derived from property (20) and the fact that $a \leq b$ for $a, b \in L$ implies $c \rightarrow_{S} a \leq c \rightarrow_{S} b$ for $c \in L^{\star}$.

Theorem 2.13. If system (30) is solvable then

$$
A_{i} \triangleleft_{S} \check{R}_{S} \subseteq B_{i}
$$

Sketch of the proof: Due to properties (18)-(19) we can prove (37) in a similar way to the proof of (29). In particular, we first prove $\check{R}_{S} \subseteq \uparrow R$. Then, based on the assumption that $\left(A_{i} \triangleleft_{S} R\right)(y)=B_{i}(y)$ is a value in $L$, we may prove that for $i=1, \ldots, m$ and $y \in Y,\left(A_{i} \triangleleft_{S} R\right)(y)$ is smaller than or equal to $\left(A_{i} \triangleleft_{S} \uparrow\right.$ $R)(y)$. When the equality holds, it can be implied that $\left(A_{i} \triangleleft_{S} \check{R}_{S}\right)(y) \leq B_{i}$. When the inequality holds, we may directly prove that $\left(A_{i} \circ_{S} \check{R}_{S}\right)(y) \leq\left(A_{i} \triangleleft_{S} R\right)(y)$ and thus, $\left(A_{i} \triangleleft_{S} \check{R}_{S}\right)(y) \leq B_{i}$.

Theorem 2.14. System (30) is solvable if and only if $\check{R}_{S}$ given in (33) is its solution, and moreover, $\uparrow R \supseteq$ $\check{R}_{S}$ for any solution $R$ of the system.

Sketch of the proof: The proof directly applies Theorem 2.12 and Theorem 2.13.

Remark 2.4. Theorem 2.14 provides a positive result of the solvability criterion of system (30). Such a result does not happen in case of applying the Sobocinski operations to the sup-T system, i.e., to system (14). To check if system (30) is solvable, it is sufficient to check whether model $\check{R}_{S}$ is its solution or not. In the case of negative, the system is not solvable. 
Similar to the sup-T system employing the Bochvar operations, system (31) is not solvable due to the appearance of the undefined value $\star$ in $A_{i}$ for $i=$ $1, \ldots, m$.

By expression

$$
\left(A_{i} \triangleleft_{K} R\right)(y)=\bigwedge_{x \in X}\left(A_{i}(x) \rightarrow_{K} R(x, y)\right),
$$

we can observe two cases which can lead to the solvability of system (32). The first case is when $\left(A_{i} \triangleleft_{K}\right.$ $R)(y) \neq \star$, which is equivalent to considering $R$ so that $R(x, y)=1$ at the values $x \in X$ such that $A_{i}(x)=\star$, and $R(x, y) \neq \star$ at the values $x \in X$ such that $A_{i}(x) \neq 0$. The second case is when $\left(A_{i} \triangleleft_{K} R\right)(y)=0$ for all $y \in Y$, which is equivalent to the existence of $x \in X$ such that $A_{i}(x)=1$ and $R(x, y)=0$. The second case implies that $\left(A_{i} \triangleleft_{K} R\right)(y)=0$ for all $y \in Y$. If model (35) is taken into account then in order to satisfy the first case, $B_{i}(y)=1$ for all $y \in Y$, and in order to satisfy the second case, $B_{i}(y)=0$ for all $y \in Y$.

Example 2.1. Let us demonstrate the possibility of having a solvable system of fuzzy relational equations when the antecedents contain undefined values by a simple example as follows. We consider a set $X$ of four criterions for buying an appartment: $x_{1}=$ "near to the workplace", $x_{2}=$ "good quality", $x_{3}=$ "good price" and $x_{3}=$ "located in a safe area", and a set $Y$ of $y=$ "decision degree" to buy it. Assume that there are three inputs $A_{1}=(0.5,0.7, \star, 1)$ and $A_{2}=(0.3,1,0.4, \star), A_{3}=(1, \star, 0.7,0.2)$ and three outputs $B_{1}(y)=0.9, B_{2}(y)=0.6$ and $B_{3}(y)=0.4$. There, * stands for the information we do not know in advance. It is posible to consider a system of three rules:

$$
\text { If } X \text { is } A_{i} \text { then } Y \text { is } B_{i}, i=1,2,3 \text {. }
$$

Let us make the illustration on the use of the lower estimation operations. We can compute the models $\hat{R}_{\ell}$ and $\check{R}_{\ell}$ :

$$
\begin{aligned}
\hat{R}_{\ell} & =(0.4,0.6,0.7,0.9), \\
\check{R}_{\ell} & =(0.4,0.6,0.9,0.9) .
\end{aligned}
$$

These models are then solutions of system (5) and system (26) respecitively. Indeed, it can be checked that $A_{i} \circ_{\ell} \hat{R}_{\ell}=B_{i}$ and $A_{i} \triangleleft_{\ell} \check{R}_{\ell}=B_{i}, i=1,2,3$.

\section{Conclusions and future work}

The solvability criterions of the systems of fuzzy relational equations employing undefined values appearing in the input fuzzy sets have been approached. To get such criterions, the models having similar shapes to the implicative model and Mamdani-Assilian model have been built. In details, the undefined values in the models regarding the sup- $\mathrm{T}$ system are lowered to 0 by applying the external operation $\downarrow$ and such values in the models regarding the inf- $\mathrm{R}$ system are increased to 1 by using operation $\uparrow$. Four sets of operations, namely, the Bochvar operations, the Sobociński operations, the Kleene operations, and the lower estimation operations have been approached for studying the solvability of the systems. The results show that, under some additional assumptions, the use of the lower estimation operations and the Sobociński operations may lead to solvable systems. As the lower estimation operations are designed for modeling missing values and the Sobociński ones are usually used for modeling indeterminable values, we may have an appropriate choice when resolving the systems regarding each type of undefined values. In the case of the input fuzzy sets contain both types of missing and indeterminable values, we have not had any conclusion for the solvability yet. Let us leave this issue for future work. This article serves as a first step toward studying the systems of fuzzy relational equations allowing the occurrence of undefined values. Such systems are important in fuzzy control and approximate reasoning due to their compatibility with the real-world application where the absence of some input information is very frequent.

In the upcoming steps, we plan to study the solvability of such systems of fuzzy relational equations, however, the consequents are taken into account as partial fuzzy sets as well. Moreover, other algebras, e.g., Dragonfly algebra [23] will also be considered for the investigation.

\section{References}

[1] L. Běhounek, M. Daňková, Towards fuzzy partial set theory, in: International Conference on Information Processing and Management of Uncertainty in Knowledge-Based Systems, Vol. 611 of Communications in Computer and Information Science, Springer, Eindhoven, The Netherlands, 2016, pp. 482-494.

[2] L. Běhounek, V. Novák, Towards fuzzy partial logic, in: 2015 IEEE International Symposium on Multiple-Valued Logic (ISMVL), IEEE, 2015, pp. 139-144.

[3] R. Bělohlávek, J. W. Dauben, G. J. Klir, Fuzzy logic and mathematics: a historical perspective, Oxford University Press, 2017.

[4] D. A. Bochvar, M. Bergmann, On a three-valued logical calculus and its application to the analysis 
of the paradoxes of the classical extended functional calculus, History and Philosophy of Logic 2 (1-2) (1981) 87-112.

[5] N. Cao, M. Štěpnička, Fuzzy relation equations with fuzzy quantifiers, in: Advances in Fuzzy Logic and Technology 2017, Springer, 2017, pp. 354-367.

[6] N. Cao, M. Štěpnička, Compositions of partial fuzzy relations, in: Information Processing and Management of Uncertainty in Knowledge-Based Systems, (IPMU'18), Vol. 855 of Communications in Computer and Information Science, Springer, 2018, pp. 187-198.

[7] N. Cao, M. Štěpnička, Compositions of partial fuzzy relations employing the lower estimation approach, in: The 10th International Conference on Knowledge and Systems Engineering (KSE 2018), IEEE, HCM, Vietnam, 2018, pp. 146-151.

[8] M. E. Cornejo, D. Lobo, J. Medina, On the solvability of bipolar max-product fuzzy relation equations with the product negation, Journal of Computational and Applied Mathematics, Elsevier (2018).

[9] M. Daňková, Quantification over undefined truth values, in: International Conference on Information Processing and Management of Uncertainty in Knowledge-Based Systems, Springer, 2018, pp. 199-208.

[10] M. Daňková, Fuzzy relations and fuzzy functions in partial fuzzy set theory, in: 10th Conference of the European Society for Fuzzy Logic and Technology, EUSFLAT 2017 and 16th International Workshop on Intuitionistic Fuzzy Sets and Generalized Nets, IWIFSGN 2017, Vol. 641 of Advances in Intelligent Systems and Computing, Springer, Warsaw, Poland, 2018, pp. 563-573.

[11] B. De Baets, Analytical solution methods for fuzzy relational equations, in: D. Dubois, H. Prade (Eds.), The Handbook of Fuzzy Set Series Vol. 1, Academic Kluwer Publ., Boston, 2000, pp. 291-340.

[12] J. Díaz-Moreno, J. Medina, E. Turunen, Minimal solutions of general fuzzy relation equations on linear carriers. an algebraic characterization, Fuzzy Sets and Systems 311 (2017) 112-123.

[13] J. C. Díaz-Moreno, J. Medina, E. Turunen, Minimal solutions of general fuzzy relation equations on linear carriers. an algebraic characterization, Fuzzy Sets and Systems 311 (2017) 112-123.
[14] M. Fitting, Kleene's logic, generalized, Journal of Logic and Computation 1 (6) (1991) 797-810.

[15] S. Freson, B. D. Baets, H. D. Meyer, Linear optimization with bipolar maxmin constraints, Information Sciences 234 (2013) 3-15.

[16] M. Gavalec, K. Zimmermann, Duality of optimization problems with generalized fuzzy relation equation and inequality constraints, Information Sciences 234 (2013) 64-70.

[17] F. Klawonn, Fuzzy points, fuzzy relations and fuzzy functions, in: V. Novák, I. Perfilieva (Eds.), Discovering the World with Fuzzy Logic, Springer, Berlin, 2000, pp. 431-453.

[18] G. Klir, B. Yuan, Fuzzy Sets and Fuzzy Logic, Prentice Hall, New Jersey, 1995.

[19] E. H. Mamdani, S. Assilian, An experiment in linguistic synthesis with a fuzzy logic controller, Int. J. Man-Mach. Stud. 7 (1975) 1-13.

[20] L. Nosková, Systems of fuzzy relation equation with inf- $\rightarrow$ composition: solvability and solutions, J. of Electrical Engineering 12(s) (2005) 69-72.

[21] I. Perfilieva, S. Lehmke, Correct models of fuzzy if-then rules are continuous, Fuzzy Sets and Systems 157 (2006) 3188-3197.

[22] E. Sanchez, Resolution of composite fuzzy relation equations, Information and control 30 (1976) $38-48$.

[23] M. Štěpnička, N. Cao, L. Běhounek, M. Burda, A. Dolný, Missing values and dragonfly operations in fuzzy relational compositions, International Journal of Approximate Reasoning submitted.

[24] M. Štěpnička, B. De Baets, L. Nosková, Arithmetic fuzzy models, IEEE Transactions on Fuzzy Systems 18 (2010) 1058-1069.

[25] X.-G. Zhou, X.-P. Yang, B.-Y. Cao, Posynomial geometric programming problem subject to maxmin fuzzy relation equations, Information Sciences 328 (2016) 15-25. 